\title{
Prophylactic (cold-chain independent) vaccination facilitated by microneedle patch of reinforced polymer with vaccine loaded silk nanoparticles
}

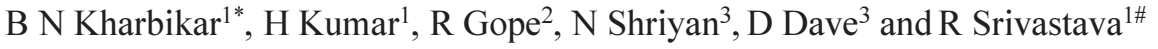 \\ ${ }^{1}$ Indian Institute of Technology Bombay, Department of Biosciences and Bioengineering, INDIA \\ ${ }^{2}$ Indian Institute of Technology Bombay, Department of Mechanical Engineering, INDIA \\ ${ }^{3}$ D Y Patil University, School of Biotechnology and Bioinformatics, INDIA \\ Email:* kharbikar.bhushan.n@iitb.ac.in,\# rsrivasta@iitb.ac.in
}

In the recent times, we have seen the outbreak of H1N1, Ebola, Zika etc. Vaccination is the critical component for the preparedness against such pandemic or epidemic. Also, regular mass immunization is required for many vaccine preventable diseases.

Such prophylactic vaccination would benefit from improved immunogenicity, elimination of cold chain, reducing need of trained personnel, bringing down pain and patient discomfort during vaccination, low cost and simplified logistic.

Here we introduce silk nanoparticles encapsulated vaccine system reinforced selfdisabling dissolving polymer microneedles patch (Figure 1).

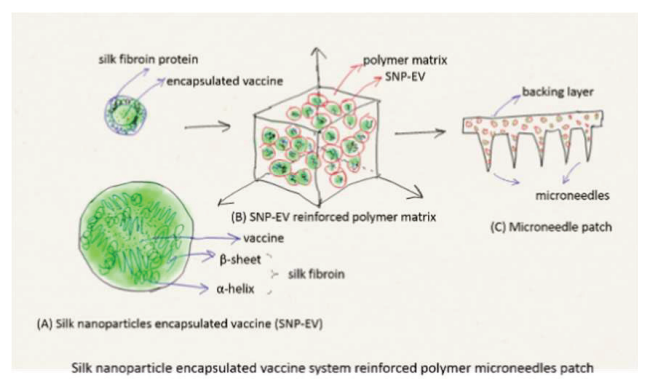

Figure 1: Silk nanoparticle encapsulated vaccine system reinforced polymer microneedles patch

This targets the large number of epidermal Langerhans cells and dermal dendritic cell for greater immunogenicity by means of epidermal vaccination using microneedle patch. Thus, it requires fewer doses to elicit antibody titers. Correspondingly, microneedles do not penetrate deeper than the epidermal skin layer with a typical thickness of $120 \mu \mathrm{m}$, to contact nerves and blood vessels and therefore, they are painless and less associated with the risks of device contamination guarantying patient comfort. No special training or skills are required hence can be self-administered. Thus, it takes care of dependency on skilled healthcare worker.

We addresses the mechanical fragility of the polymeric microneedle structures by reinforcing them using silk nanoparticles which has inherent mechanical robustness due to highly networked $\beta$-sheet structures. In addition to that, enhancement of mechanical strength is endorsed to larger surface area, which transfers the load from polymer matrix to silk nanoparticles.

Vaccine is encapsulated in the synthesized silk nanoparticles which provides thermal shield to vaccine and prevent it from getting denatured hence preventing its bioactivity. This is by the virtue of highly thermodynamically stable nature of silk, which is attributed to extensive physical crosslinking, highly hydrophobic protein and high glass transition temperature. This reduces the dependency on cold chain maintenances and eases the logistic as well. The proposed system is simulated, synthesized, fabricated and characterized. The results suggest that it brings in the complete set of attributes addressing all the challenges which impacts the prophylactic vaccination.

Thus, the novel system revolutionizes the vaccination with multifold increase in the effectiveness and coverage of vaccination.

Keywords: silk nanoparticles, vaccine delivery, cold chain independent, microneedle, immunogenicity, epidermal

\section{References}

1. Sullivan et.al," Nat Med, vol. 16, pp. 915-920, 2010 .

2. Seib et.al, Advanced Healthcare Materials, vol. 2, pp. 1606-1611, 2013. 